OPEN ACCESS

Edited by:

Tinggui Chen,

Zhejiang Gongshang University, China

Reviewed by:

Yunfang Peng,

Shanghai University, China

Xing $\mathrm{Xu}$,

Zhejiang University of Science and

Technology, China

*Correspondence:

Du Jiang

jiangdu@wust.edu.cn

Bo Tao

taoboq@wust.edu.cn

Guozhang Jiang

whigz@wust.edu.cn

Specialty section:

This article was submitted to

Bionics and Biomimetics,

a section of the journal

Frontiers in Bioengineering and

Biotechnology

Received: 12 October 2021

Accepted: 17 December 2021

Published: 10 January 2022

Citation:

Liu $X$, Jiang $D$, Tao $B$, Jiang $G$, Sun $Y$,

Kong J, Tong $X$, Zhao $G$ and Chen $B$

(2022) Genetic Algorithm-Based

Trajectory Optimization for Digital

Twin Robots.

Front. Bioeng. Biotechnol. 9:793782.

doi: 10.3389/fbioe.2021.793782

\section{Genetic Algorithm-Based Trajectory Optimization for Digital Twin Robots}

\author{
Xin Liu ${ }^{1,2}$, Du Jiang ${ }^{1,3 *}$, Bo Tao ${ }^{1,4 *}$, Guozhang Jiang ${ }^{1,2,4 *}$, Ying Sun ${ }^{1,2}$, Jianyi Kong ${ }^{1,2,3}$, \\ Xiliang Tong ${ }^{1}$, Guojun Zhao ${ }^{1,4}$ and Baojia Chen ${ }^{5}$ \\ ${ }^{1}$ Key Laboratory of Metallurgical Equipment and Control Technology of Ministry of Education, Wuhan University of Science and \\ Technology, Wuhan, China, ${ }^{2}$ Research Center for Biomimetic Robot and Intelligent Measurement and Control, Wuhan University \\ of Science and Technology, Wuhan, China, ${ }^{3}$ Hubei Key Laboratory of Mechanical Transmission and Manufacturing Engineering, \\ Wuhan University of Science and Technology, Wuhan, China, ${ }^{4}$ Precision Manufacturing Research Institute, Wuhan University of \\ Science and Technology, Wuhan, China, ${ }^{5}$ Hubei Key Laboratory of Hydroelectric Machinery Design \& Maintenance, Three \\ Gorges University, Yichang, China
}

Mobile robots have an important role in material handling in manufacturing and can be used for a variety of automated tasks. The accuracy of the robot's moving trajectory has become a key issue affecting its work efficiency. This paper presents a method for optimizing the trajectory of the mobile robot based on the digital twin of the robot. The digital twin of the mobile robot is created by Unity, and the trajectory of the mobile robot is trained in the virtual environment and applied to the physical space. The simulation training in the virtual environment provides schemes for the actual movement of the robot. Based on the actual movement data returned by the physical robot, the preset trajectory of the virtual robot is dynamically adjusted, which in turn enables the correction of the movement trajectory of the physical robot. The contribution of this work is the use of genetic algorithms for path planning of robots, which enables trajectory optimization of mobile robots by reducing the error in the movement trajectory of physical robots through the interaction of virtual and real data. It provides a method to map learning in the virtual domain to the physical robot.

Keywords: genetic algorithm, mobile robot, digital twin, virtual model, trajectory optimization

\section{INTRODUCTION}

Mobile robots are an important branch of the industrial robot family, and their demand accounts for about $30 \%$ of the demand for industrial robots (Niloy et al., 2021). Mobile robots integrate the comprehensive application technology of mechanical, computer, artificial intelligence and other disciplines, which can effectively improve the efficiency of industrial production, reduce the labor intensity of workers, and increase economic benefits (Duan et al., 2021). Mobile robots use sensors to sense the environment and achieve autonomous movement in complex environments according to rational algorithms (He et al., 2019; Chen et al., 2021a). Path planning is the planning of a collisionfree path from the current position to the target position under the constraints using the relevant algorithms (Hu et al., 2019; Huang et al., 2021). Path planning algorithms include genetic algorithms, ant colony algorithms, etc. These algorithms are used in different situations due to different computational principles (Li et al., 2019a; Jiang et al., 2021a). Most of the current research on mobile robots uses improved algorithms to reduce their optimal path length or the number of iterations, and there is a lack of research on error control during the actual motion of the robot (Li et al., 2019b; Bai et al., 2021). In practice, the robot cannot follow the planned path to the target position during the movement due to the influence of environmental factors or the error in the 
coordination of various parts of the robot (Chen et al., 2021b; Chen et al., 2021c). At the same time, the lack of data feedback from most robots during movement makes it impossible to determine where errors occur and to correct the robot's work path, making it impossible for the robot to work in workplaces with high operational accuracy (Yu et al., 2019; Luo et al., 2020).

With the introduction of national-level manufacturing development strategies such as the U.S. Industrial Internet, Germany's Industry 4.0 and Made in China 2025, smart manufacturing has become a common trend and goal for global manufacturing development (Jiang et al., 2019a; Cheng et al., 2021). As a key technology to realize the concept and goal of smart manufacturing, digital twin has received wide attention from academia and is being applied in more and more industrial fields (Hao et al., 2021; Jiang et al., 2021b). Digital twin is a technical means to realize the information interaction between the physical world and the virtual world, which creates virtual models of physical entities digitally, simulates the operation of physical entities in the real environment with the help of actual data, and dynamically optimizes the working state of physical entities (Tao et al., 2019; Lu et al., 2020; Jiang et al., 2021a). As a technology that makes full use of models and data and integrates multiple disciplines, the digital twin is oriented to the whole product lifecycle process, playing the role of a bridge and link between the physical world and the information world to provide more real-time, efficient and intelligent services (Jiang et al., 2019c; Huang et al., 2020; Liao et al., 2021). Digital twin-based precision distribution for production logistics is a combined application of digital twin technology and mobile robots ( $\mathrm{Li}$ et al., 2019c; Li et al., 2020). Production logistics, including internal logistics and external logistics, is the key to ensure normal production, improve production efficiency and reduce product costs (Liu J et al., 2021; Yang et al., 2021). Digital twin production logistics refers to a new production logistics operation mode driven by twin data, through real mapping of physical entities and virtual models, real-time interaction and closed-loop control, to achieve task combination optimization, transportation path planning and transportation process control of production logistics, so as to achieve seamless and intelligent production process logistics (Sun et al., 2020b; Liu et al., 2021b).

In this paper, we develop a mobile robot based on digital twin technology, build a virtual environment in Unity, and complete the path planning of the virtual model using genetic algorithm. A communication architecture between the virtual model and the physical robot is proposed to complete the data interaction between the robot and the virtual model via Bluetooth, which achieves dynamic optimization of the physical robot movement process and improve the accuracy of the robot's movement trajectory. In contrast to the work done by others, the focus of our work is to emphasize the real-time mastery and correction of errors generated during robot movement, and to achieve the reduction of errors in physical entities through the interaction of virtual models and physical entities. There are three contributions of this paper.

1) A robot that can move autonomously was developed.

2) A digital twin of the mobile robot was built in Unity.
3) The path planning of the robot was implemented in a virtual environment based on genetic algorithm.

4) A trajectory optimization method for a mobile robot was proposed. The trajectory error of the robot is gradually reduced by virtual-real interaction.

The rest of this paper is organized as follows. Reviewed some of the research done by domestic and international scholars on mobile robots and digital twins in Section 2. Section 3 describes the virtual environment of the robot and the genetic algorithmbased path planning in the virtual environment. Section 4 presents the experiments of digital twin-based mobile robot trajectory optimization and summarizes the experimental results. Section 5 concludes the paper with summary and future research directions.

\section{RELATED WORK}

Mobile robots have the characteristics of high efficiency, wide working range and convenient operation, etc. With the continuous enrichment of computer control theory and deep learning and other related theories, the requirements for the movement accuracy of robots have gradually increased Material and Methods (Huang et al., 2019; Miao et al., 2021). Robots with high motion accuracy can improve efficiency and avoid wasting resources when moving with high accuracy requirements, especially when completing transportation tasks in high-risk production areas (Ma et al., 2020; Sun et al., 2020a; Liu et al., 2021a). HUR Sung Wook et al. propose a new approach to efficient trajectory optimization that exploits the fact that the dynamics of a deterministic system is uniquely determined by the initial state and control over the time horizon of interest (Hur et al., 2021). Hu proposed a method based on content image retrieval to identify obstacles on the robot's running path $(\mathrm{Hu}$, 2021). Fu et al. analyzed the local minima problem and the target unreachability problem which are easy to occur in the artificial potential field method, and solved the local minima problem of the artificial potential field method better by introducing the virtual obstacle model (Fu et al., 2021). Huo et al. proposed an optimal fuzzy logic obstacle avoidance algorithm for implementing motion obstacle avoidance of mobile robots (Huo and Wang 2021). Zheng et al. proposed a laser-based person detection and obstacle avoidance algorithm for differential drive robots applied to a handling robot to transport materials along a reference path in the hospital field (Zheng et al., 2021). Luka Petrović et al. proposed a new trajectory planning algorithm using stochastic optimization in order to find a continuous-time Gaussian process for collision-free trajectory generation (Petrović et al., 2020). Deng et al. proposed a multiobstacle path planning and optimization method that uses convex packages to optimize the base obstacles and obtain the corresponding set of base obstacle points, and uses cubic bezier curves to smooth the path to fit the kinematic model of the robot (Deng et al., 2021).

The concept of digital twin was proposed by Prof. Michael Grieves, who showed in his paper that virtual models and related 
subsystems are constructed to represent physical entities in a virtual information space and establish two-way dynamic connections through data from physical devices in real space, but due to technical limitations at that time, the concept of digital twin did not gain much attention (Tan et al., 2020; Sun et al., 2021). In 2011, NASA applied the digital twin concept to the Apollo project to construct virtual bodies of space vehicles in virtual information space, and through the observation and analysis of the virtual bodies, the prediction and maintenance of the flight status of space vehicles were realized, and then the digital twin technology began to attract attention (Tao et al., 2018; Liu Y et al., 2021a). Many internationally renowned companies have already started to explore the application of digital twin technology in product design, manufacturing and service (Sun et al., 2020c; Tao et al., 2021). In product design, for the innovative design of complex products, Dassault has established a $3 \mathrm{D}$ experience platform based on the digital twin, which uses the information from user interaction to continuously improve the product design model in the information world and to implement it into the physical product improvement (Sun et al., 2020d; Fu et al., 2021). In manufacturing, Siemens has built a production system model that integrates manufacturing processes based on the digital twin concept, formed a virtual enterprise based on the model and an enterprise mirror based on automation technology, and carried out application validation in the production process of Siemens industrial equipment: Nanobox PC. In terms of product service, PTC has made digital twin a key aspect of intelligent and connected products and is committed to establishing a real-time connection between the virtual world and the real world, enabling predictive maintenance of products and providing customers with efficient product after-sales service and support.

The digital twin technology mainly includes important parts such as the creation of virtual models, the collection of actual data, and the data interaction between physical entities and virtual models. The determination of model parameters, the fit constraints between components and the accuracy of the model are the key issues and difficult problems of the virtual model. Currently, research has been conducted in the framework and process of digital twin modeling, and there are many software for modeling, but there is a lack of a complete set of modeling theory and modeling process (Jones et al., 2020; Liu et al., 2020). Digital twin technology requires a high level of real-time data, and in most occasions automated data collection is used, which relies on the use of devices with good communication conditions and various types of sensors. Data interaction between physical entities and virtual models is the key to the implementation of digital twin technology, but there is little research related to the interaction and collaboration between machines and services (Zhuang et al., 2017; Guo et al., 2021; Ruzsa, 2021). Verner et al. developed a system of reinforcement learning scenarios in which humanoid robots learn the protocols required to lift weights of unknown mass by exploring state space. To speed up the process of physical training, these experiments were performed in a virtual space, simulated in a digital twin, where the parameters obtained from simulation learning were mapped onto the physical robot (Verner et al., 2018). Huang et al. demonstrated a linear tracking robot trained in digital twin mode in a virtual space (Huang et al., 2019). Matulis et al. present a method for creating and training a digital twin robot for a robotic arm. The project demonstrates that a trained robot can perform a given task even if it is currently in a state it has never been in before (Matulis and Harvey, 2021). Liu et al. proposed a digital-driven machining quality tracking and dynamic control method, which effectively solved the problems of low efficiency of quality problem traceability, poor timeliness and unpredictability of quality control in machining process (Liu Y et al., 2021b).

The application of digital twin technology in logistics and distribution is one of the future directions of digital twin, which realizes the accurate distribution of goods through the control of the distribution process of mobile robots. Currently, most of the domestic research on mobile robots considers the robot's movement path planning. From the overall effect, the improved path planning algorithm improves the movement accuracy of the robot, but it is impossible to know the location where the movement trajectory error occurs and lacks feedback on the error during the robot movement (Liu et al., 2021d). Applying digital twin technology to mobile robots and establishing the interaction between robots and virtual models can grasp the robot's operation in real time and optimize the robot's movement trajectory (Zhao et al., 2021). Digital twinbased mobile robots can be applied to applications where trajectory attractions are demanding, such as power station inspection, space exploration, etc.

\section{MATERIALS AND METHODS}

This section describes the general approach and workflow for improving the trajectory accuracy of mobile robots, including the setup of virtual environments and genetic algorithm-based path planning. Figure 1 provides a functional overview of the digital twin robot virtual-reality interaction method. The robot entity is controlled by STM32 microcontroller, the orthogonal code disk determines the spatial coordinates of the robot and creates a virtual model of the robot in untiy. STM32 establishes communication with Unity's virtual serial port to achieve data interaction between the physical entity and the virtual model.

\subsection{Physical Robot}

The physical entity is objective and usually consists of control subsystems, power subsystems, actuation subsystems, etc. and accomplishes specific tasks through collaboration among the subsystems, and its environmental data and operational status are monitored in real time by sensors deployed on the physical entity (Weng et al., 2021). The robot is built from aluminum profiles and driven by RoboMaster M2006 DC brushless motors. The robot weighs about $13 \mathrm{~kg}$ and can achieve movements such as straight line movement, lateral movement, turning and other movements. The motors provide a maximum speed of up to $500 \mathrm{rpm}$, a maximum 


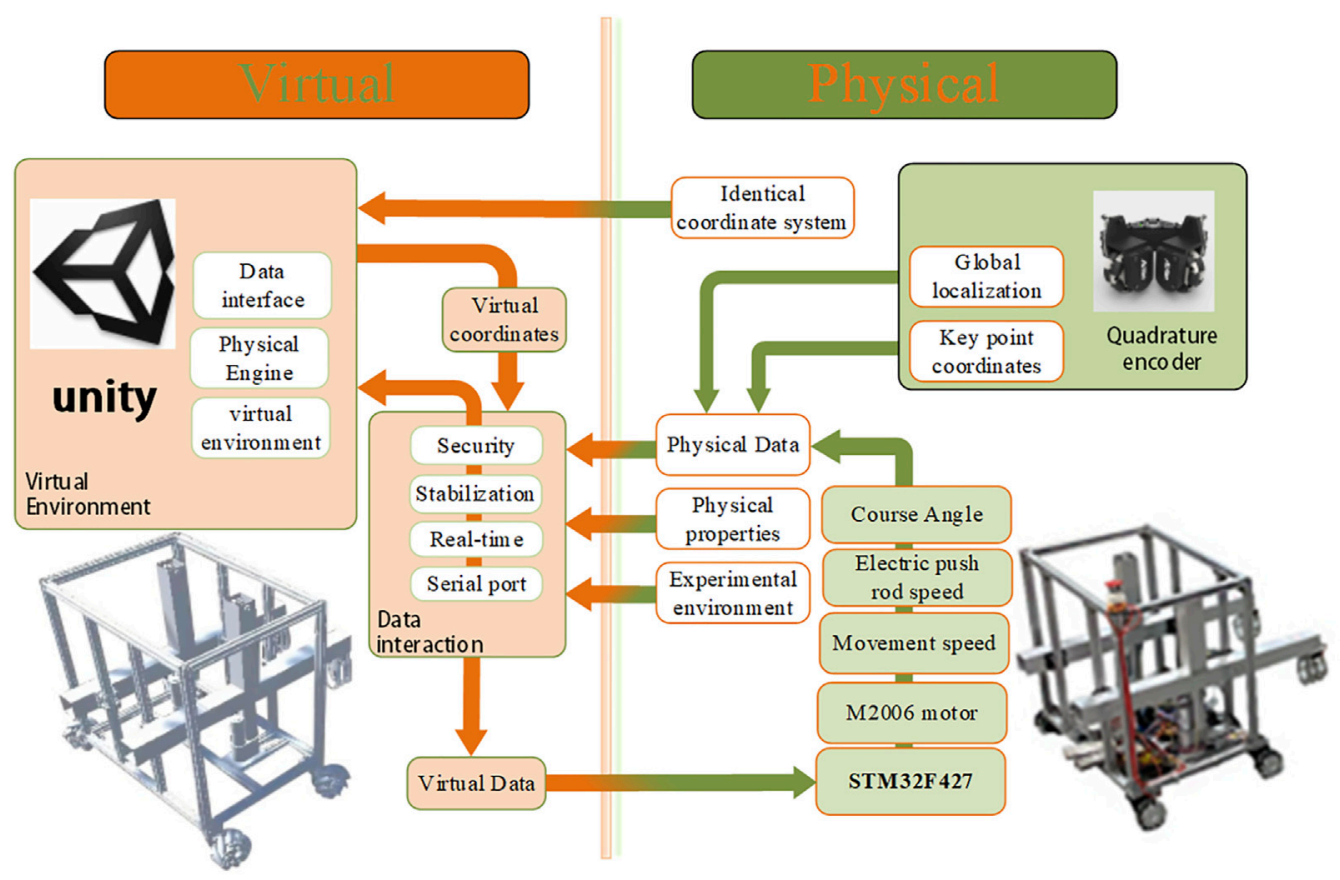

FIGURE 1 | Framework for interaction between virtual models and physical entities.

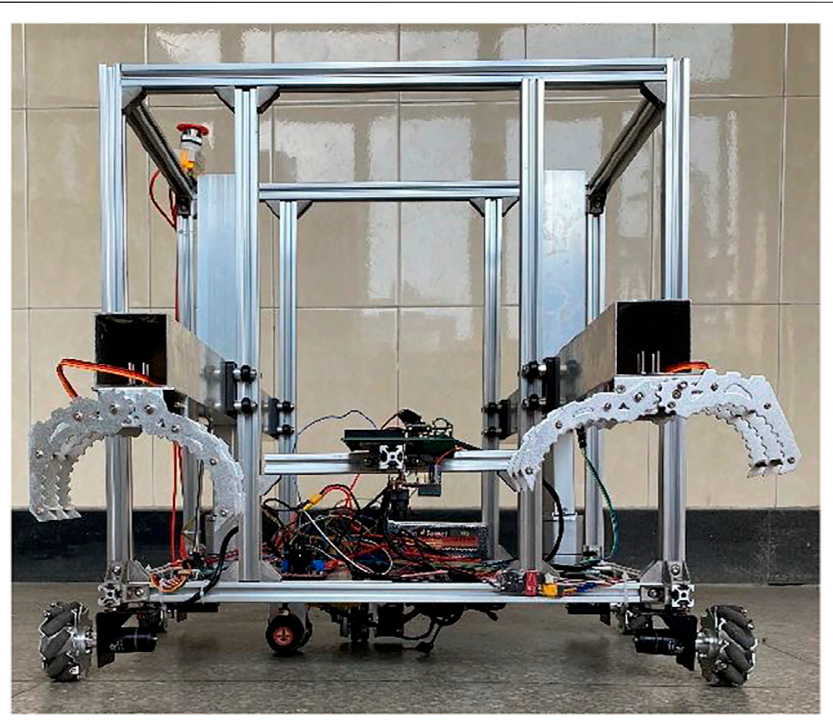

FIGURE 2 | Physical structure of the robot.

sustained torque of $1,000 \mathrm{mN} \mathrm{m}$, and a maximum sustained output power of $44 \mathrm{~W}$. Each motor is controlled by a 32-bit microprocessor STM32 board. The STM32 board controls the M2006 motor to drive the robot movement via the c610 electronic speed controller, using an orthogonal encoder and gyroscope to determine the robot's position coordinates. The orthogonal encoder has two directions, $\mathrm{X}$ and $\mathrm{Y}$, corresponding to one coordinate on the plane. By giving the coordinates, it can realize the fixed-point movement of the robot, and its combination with the gyroscope can realize the correction of the running trajectory by controlling the number of revolutions of the motors. The $\mathrm{L} 298 \mathrm{~N}$ module controls the electric actuators and mechanical jaws to grasp the object. The physical entity of the robot is shown in Figure 2.

\subsection{Virtual Environment Construction}

The virtual model is a digital mirror of the physical entity and mainly includes four layers of models: geometry, behavior, interaction, and association. Geometric models mainly describe geometric relationships such as size and shape (Xiao et al., 2021). Behavioral models analyze expected behavior, actual behavior, and random behavior. Interaction model refers to the data interaction, behavior interaction, information interaction between virtual model and physical entity (Liu Y et al., 2021c; Yun et al., 2021). The association model describes the interactions between the geometric model, the behavioral model, and the interaction model.

Unity was chosen to complete the construction of the virtual model, the motion control of the model, and the development of the experimental scenes. In Unity, a robot movement scene is built as shown in Figure 3, which includes a virtual model of the robot, two cylindrical obstacles, a white goods stacking area, and handing objects. When constructing the virtual model, it is necessary to consider the influence of realistic factors, such as the robot's material, mass, and the robot's movement speed. The specific parameters of the experimental environment are shown in Table 1. The requirement for the robot's movement path is that the robot starts from the starting position, carries the handling objects through two obstacles, and finally reaches the white goods stacking area. The ability of the robot to 


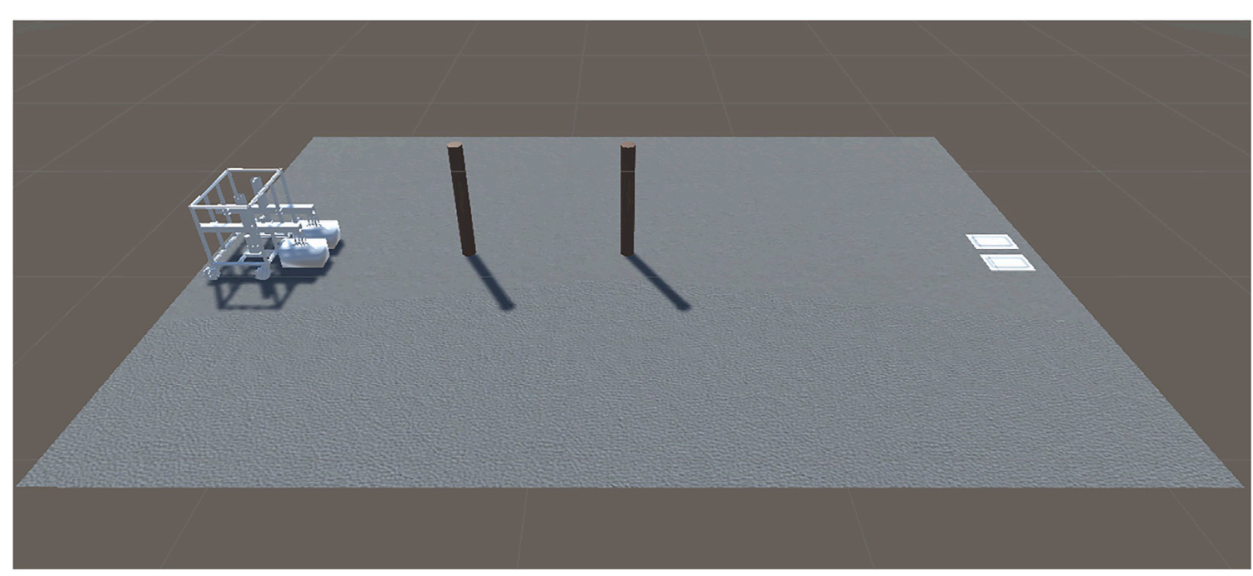

FIGURE 3 | Virtual environment.

TABLE 1 | Experimental environment parameters.

\section{Facility name}

Grey base plate

Obstacles

Stacking area

Handling objects
Distance between obstacles
Dimensional parameters

$3 \mathrm{~m} \times 5 \mathrm{~m}$

$\mathrm{d}=0.1 \mathrm{~m} ; \mathrm{h}=1 \mathrm{~m}$

$0.4 \mathrm{~m} \times 0.3 \mathrm{~m}$

$0.28 \mathrm{~m} \times 0.16 \mathrm{~m} \times 0.15 \mathrm{~m}$ $1.2 \mathrm{~m}$ place the load in the stacking area is a criterion for determining whether the robot is experiencing trajectory errors. There is a maximum speed limit on the movement of the physical robot and a height limit on the rise of the electric actuators, which must be captured and applied to the virtual environment during the experiment.

\subsection{Genetic Algorithm-Based Path Planning}

Genetic algorithms are stochastic global search optimization methods that simulate the phenomena of replication, crossover, and variation that occur in natural selection and inheritance. Starting from an initial population, the population evolves to increasingly better regions in the search space by random selection, crossover and mutation operations to produce a group of individuals better suited to the environment, and finally converges to a group of individuals best suited to the environment to obtain a quality solution to the problem (Nazarahari et al., 2019; Sarkar et al., 2020). For path planning based on genetic algorithm, the individuals suitable for the environment are the suitable moving paths, and the one that best satisfies the conditions is obtained by random selection and crossover variation. The flow chart of virtual environment robot path planning based on genetic algorithm is shown in Figure 4.

The steps of genetic algorithm-based path planning for virtual environment robots are as follows.

1) The raster method is used to model the robot's walking space, which is represented by a square. The white grid indicates the

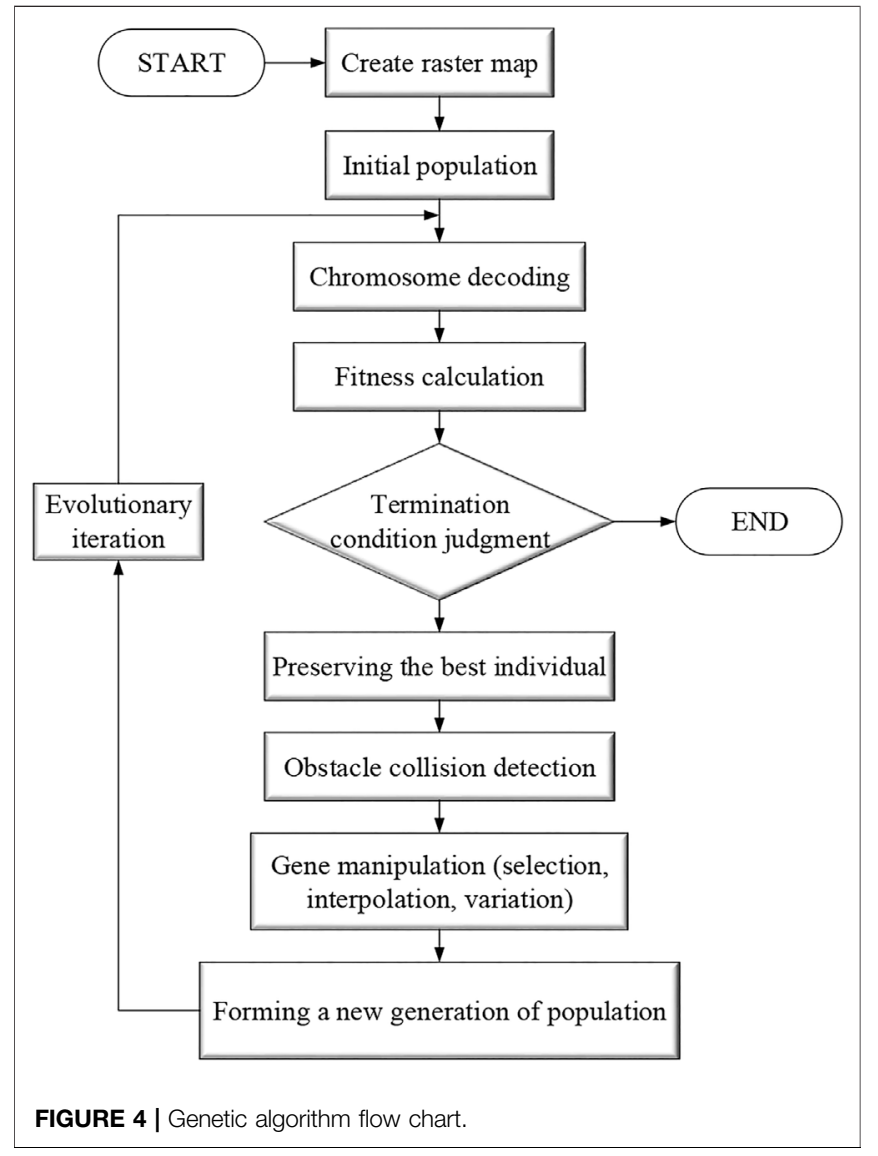

moveable area and the black grid indicates the obstacle. In this paper, the robot walking space is shown in Figure 5.

The Cartesian coordinate system is established with the first grid in the lower left corner of the map as the coordinate origin, so the coordinates of each grid can be expressed as ( $x, y)$. For example, the first grid in the bottom left corner can be represented as $(1,1)$. The number in the raster represents the number $n$. The 


\begin{tabular}{|c|c|c|c|c|c|c|c|}
\hline 7 & 42 & 43 & 44 & 45 & 46 & 47 & 48 \\
\hline 6 & 35 & 36 & 37 & 38 & 39 & 40 & 41 \\
\hline 5 & 28 & 29 & 30 & 31 & 32 & 33 & 34 \\
\hline 4 & 21 & 22 & 23 & 24 & 25 & 26 & 27 \\
\hline 3 & 14 & 15 & 16 & 17 & 18 & 19 & 20 \\
\hline 2 & 7 & 8 & 9 & 10 & 11 & 12 & 13 \\
\hline 1 & 0 & 1 & 2 & 3 & 4 & 5 & 6 \\
\hline & 1 & 2 & 3 & 4 & 5 & 6 & 7 \\
\hline
\end{tabular}

conversion formula between number and coordinate is shown in Equation 1. In this paper, the grid numbers of the starting and ending points are 3 and 45 , and the grid numbers of the obstacles are 17 and 31 , respectively.

$$
\left\{\begin{array}{l}
x=\operatorname{int}(n / 7)+1 \\
y=n \% 7+1
\end{array}\right.
$$

2) Initialize chromosomes and use floating point encoding to form populations. The purpose of initializing the population is to randomly generate multiple feasible paths, feasible paths being those that do not collide with the obstacle grid.

3) To allow the dominant individual to be preserved, define the fitness function as shown in Equation 2.

$$
f_{\text {fitvalue }}=\exp (200 / \text { dis } * \mu)-1
$$

Where dis is the distance of each individual (path), $\mu$ is the collision coefficient between the individual and the obstacles. If the path collides with the obstacle, then $\mu$ take 0 , if no collision then $\mu$ take 1 . Obviously, if the path collides with an obstacle, its fitness is 0 . It will not be inherited to the next generation, and this individual is discarded. dis is calculated using the Euclidean distance, and the calculation formula is shown in Equation 3.

$$
d i s_{i+1}=\sqrt{\left(x_{i+1}-x_{i}\right)^{2}+\left(y_{i+1}-y_{i}\right)^{2}}
$$

4) A number of individuals were selected from the population using the roulette selection method. In this method, the selection probability of each individual is proportional to its fitness value, and the higher the fitness value, the higher
TABLE 2 | Genetic algorithm parameter settings.

\begin{tabular}{lc}
\hline Parameters & Value \\
\hline Population size & 50 \\
Evolutionary algebra & 100 \\
Number of chromosomes & 5 \\
Mutation probability & 0.045 \\
Gene conversion probability & 0.1 \\
Gene crossover probability & 0.9 \\
Gene variation probability & 0.07 \\
Select Strategy & Roulette
\end{tabular}

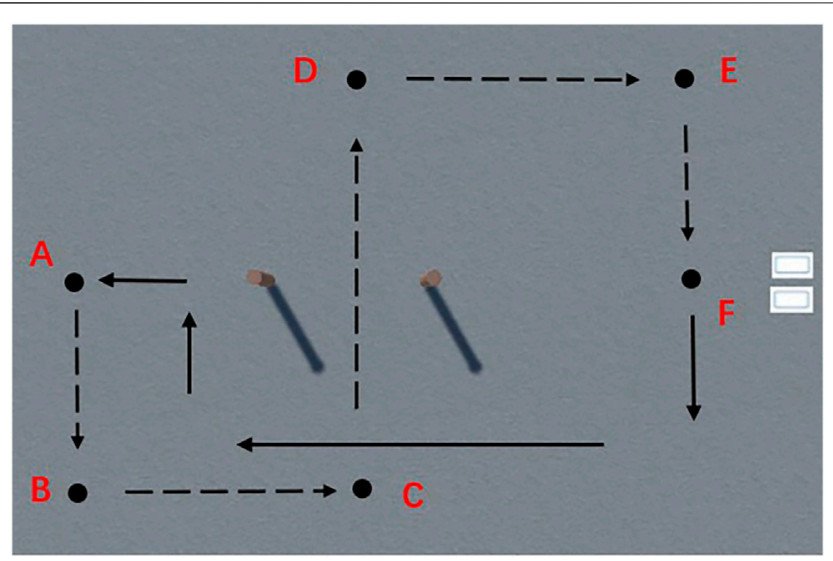

FIGURE 6 | Virtual robot movement path.

the probability of being selected. The specific steps are as follows.

1) Calculate the fitness of each individual in the population.

2) Calculate the probability of each individual being inherited into the next generation population, as shown in Equation 4.

$$
P\left(\text { dis }_{i}\right)=\frac{f_{\text {fitvalue }}\left(\text { dis }_{i}\right)}{\sum_{j=1}^{N} f_{\text {fitvalue }}\left(\text { dis }_{i}\right)}
$$

3) Calculate the cumulative probability of each individual, as shown in Equation 5.

$$
q_{i}=\sum_{j=1}^{i} P\left(d i s_{i}\right)
$$

4) Generate a uniformly distributed pseudo-random number $r$ in the interval [0,1]. If $q_{k-1}<\mathrm{r} \leq q_{k}$ is satisfied, select individual $k$.

5) A random number approach is chosen for chromosome crossover operations to form new chromosomes, as shown in Equation 6. where $a$ is the generated random number, $s_{i}$ is the child, $f a t_{i}$ and $f a t_{i-1}$ are the parents.

$$
s_{i}=(1-a) * f a t_{i}+a * f a t_{i-1}
$$

6) The uniform variation operator is selected for variation operations. The original gene values at each locus in the 


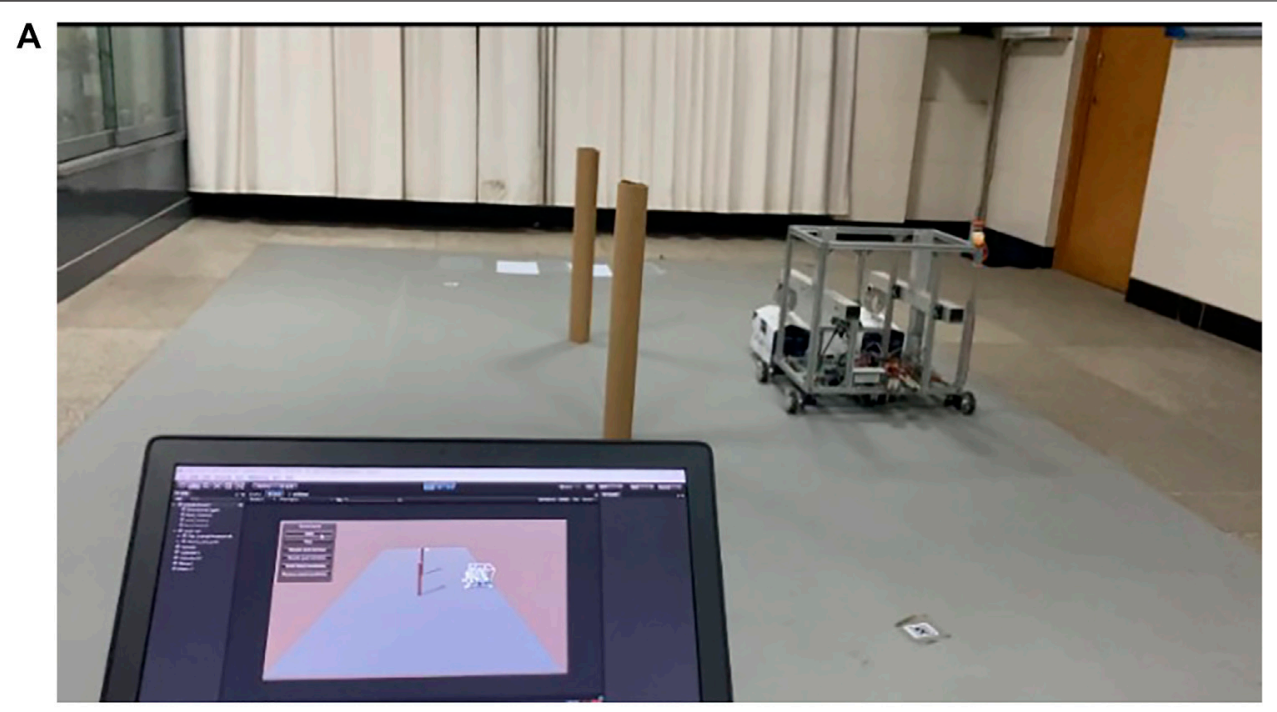

B

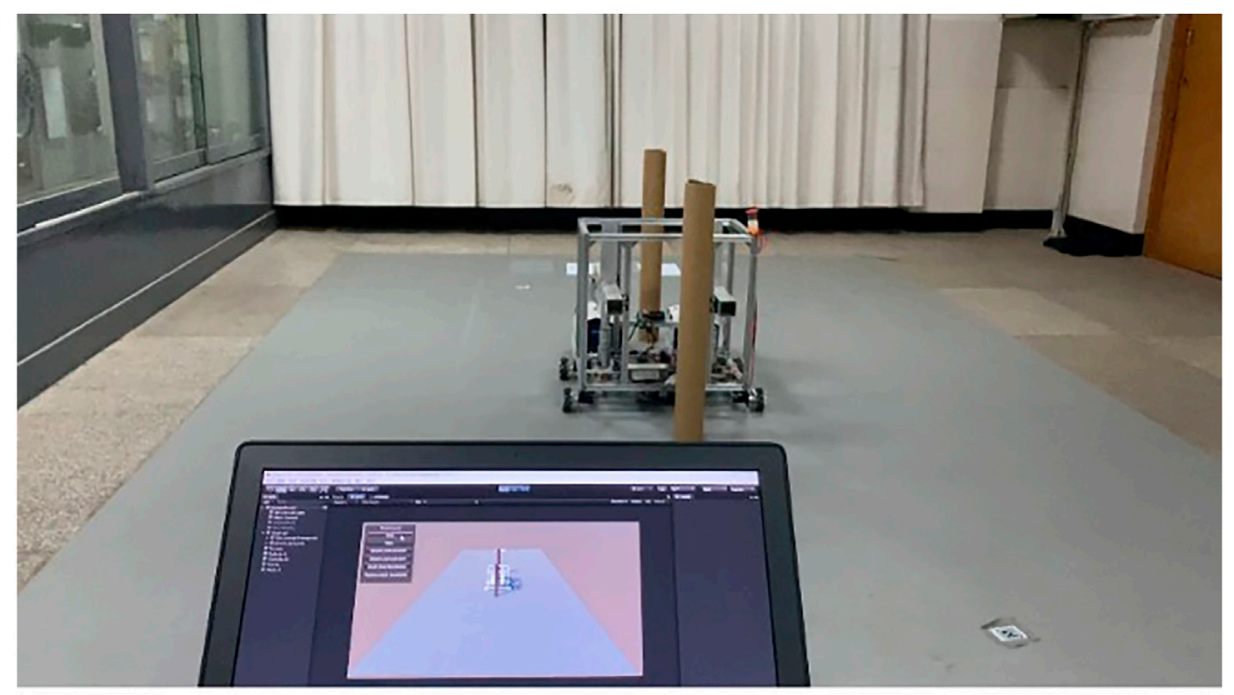

FIGURE 7 | Virtual models control the movement of physical robots. Panel (A) indicates that the robot passes the path point C; Panel (B) shows the robot passes an obstacle.

individual coding string are replaced with a random number that matches a uniform distribution within a certain range with a certain small probability.

7) Formation of new individuals of the next generation. Calculate the fitness value of the new individual.

8) Determine whether the best individuals in the new generation population meet the expected requirements, and if so, output the result and go to step (I), otherwise, return to step $(\mathrm{E})$.

9) Define two termination criteria: 1) Maximum number of iterations is 100 ; 2) Little chromosome variation and population stabilization. Before each genetic operation, determine whether the termination criterion is satisfied, and if the condition is satisfied, the optimization process ends. The specific parameters are shown in Table 2 .

According to the decoding method of genetic algorithm, the input is the coordinates of the starting point, target point and obstacles, and the output is the corresponding motion trajectory. According to the actual working conditions and task requirements of the physical robot, the motion trajectory of the robot is determined as shown in Figure 6. point $\mathrm{A}$ and point $\mathrm{F}$ denote the starting point and end point respectively, and points B, C, D and E are path critical points. The physical robot follows the dashed path from the starting point A through the obstacles to the end point $\mathrm{F}$, and then returns along the solid path. 


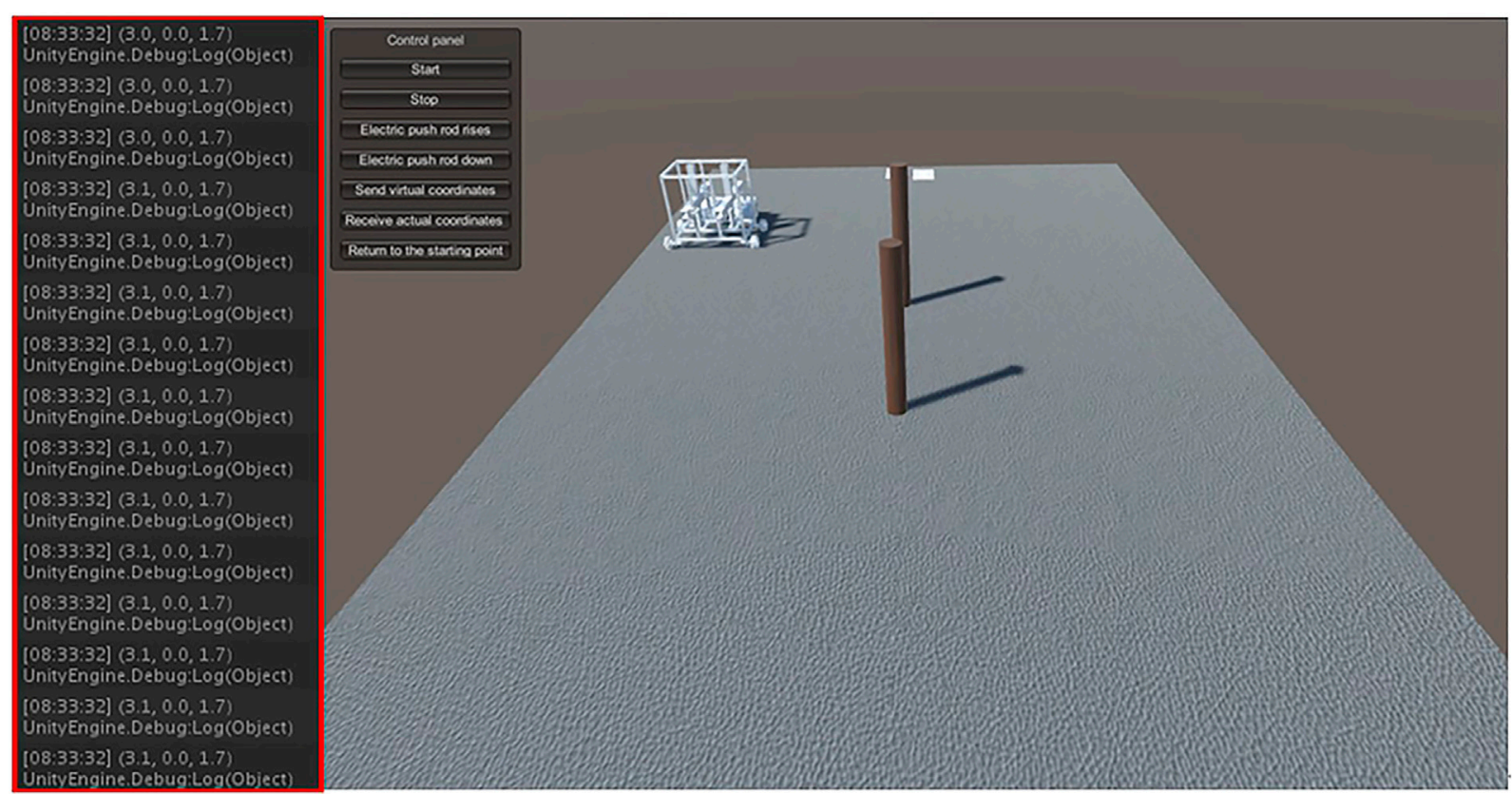

FIGURE 8 | Virtual environments receive real-time movement data from physical robot.

\section{RESULTS}

\subsection{Experimental Procedure}

In digital twin technology, the virtual model serves as a realistic mapping, simulation, and feedback correction. After several simulation tests in the virtual environment, appropriate operating parameters are determined and sent to the physical entity to realize the control of the virtual model over the physical entity (Liu J et al., 2021). Before the experiment starts, set the moving speed of the physical robot and the lifting speed of the electric actuator to constant values. Since the physical robot uses an orthogonal encoder for global positioning, the directional movement of the physical robot can be achieved by specifying the location coordinates of the target point.

The specific operation process is: establish the same global coordinate system as the virtual environment in the physical robot control system, send the coordinates of points A, B, C, D, E and F in Figure 5 to the physical robot STM32 board, then the robot can move according to the coordinates after receiving the path coordinates. The movement process of the robot is shown in Figure 7. From the figure, it is clear that the physical robot moves according to the path obtained by training in the virtual environment.

After the physical robot starts moving, the STM32 board sends the real-time coordinates of the moving path to the virtual environment. The virtual model moves in the virtual environment based on the obtained real-time coordinates, and determines the position of the robot trajectory offset by comparing it with the predefined running trajectory. The coordinates of the key points of the path are continuously adjusted according to the trajectory offset, thus gradually improving the accuracy of the actual moving trajectory of the

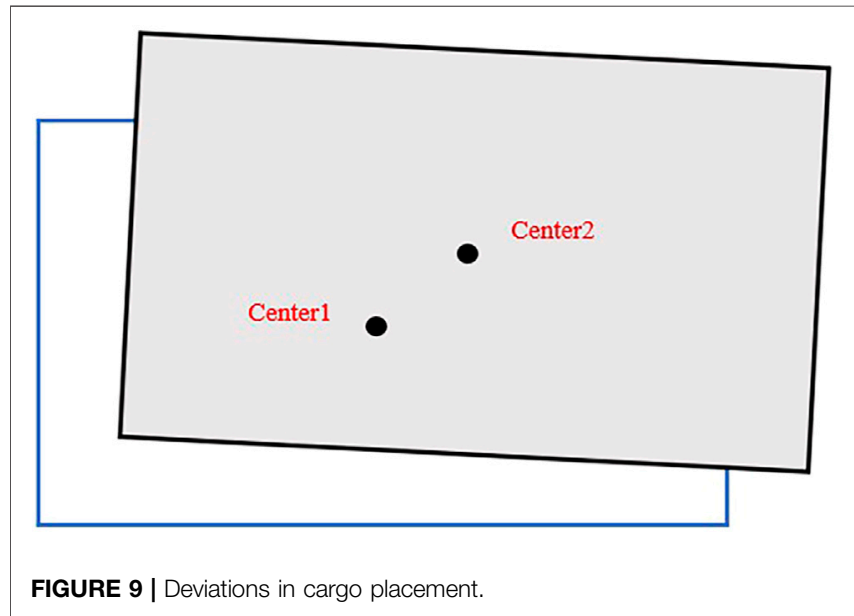

physical robot. The experimental procedure is shown in Figure 8, where the red rectangular box shows the real-time data of the physical robot movement received by the virtual environment.

\subsection{Analysis of Errors and Sensitivities}

The physical robot cannot reach the position of the specified coordinate point during the movement. The reasons for this phenomenon are measurement error, physical error, virtual error and other aspects. Measurement error refers to the dimensional measurement error and assembly error that occurs during the building process of the physical robot. Physical errors are the errors between the robot and the environment and the errors in the robot hardware, mainly the friction between the ground and the 


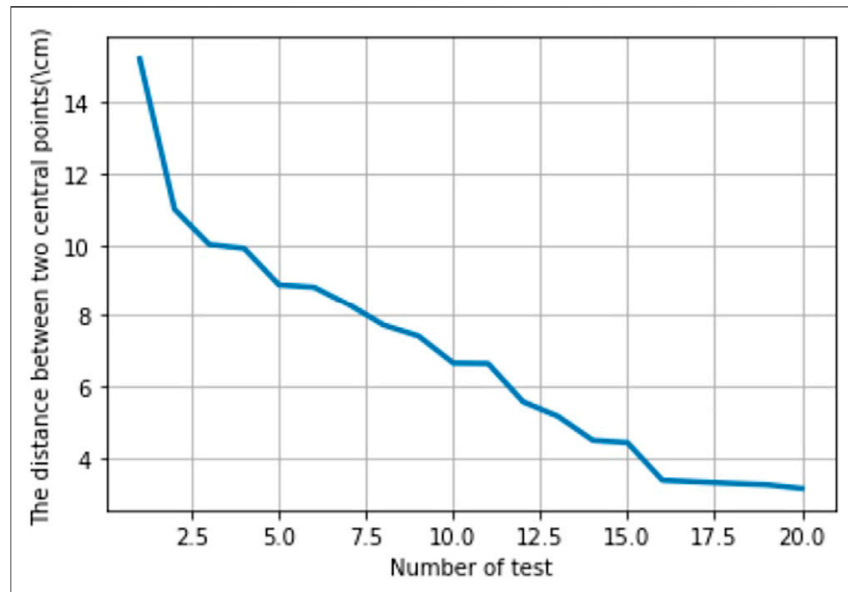

FIGURE 10 | Trend of distance between two center points.

wheels, the encoder error and the gyroscope heading angle error. Virtual errors refer to the model dimensional errors and virtual model assembly errors that occur when modeling in the virtual environment.

Sensitivity analysis is the process of finding out the most influential factor on the experimental results among many uncertainties and analyzing the degree of its influence on the experimental target. Among several types of errors affecting the accuracy of robot motion trajectory, both measurement errors and virtual errors can be artificially controlled to reduce the occurrence of errors. Physical errors are random in nature and uncontrollable. Considering several factors that generate physical errors, among them, the friction between the ground and the wheels has the greatest influence on the experimental results, so the influence of other errors is ignored and only the influence brought by the friction between the ground and the wheels is considered.

\section{Analysis of Experimental Results}

The reason for setting the stacking area is that it is difficult to observe whether the trajectory deviation occurs during the actual movement of the robot, and it can be judged whether the robot shifts during the movement by whether the robot can place the goods correctly in the stacking area. Figure 9 shows a situation where the goods are not placed correctly, from which it can be determined that the physical robot has shifted during the movement. The larger the area of cargo deviation from the stacking area, the larger the amount of robot trajectory deviation.

Center 1 is used to indicate the center of the bottom surface of the goods, and Center 2 indicates the center of the stacking area. The distance between Center1 and Center2 is used to indicate the degree of deviation of the goods not correctly placed in the stacking area, as shown in Figure 8. The experimental procedure in Section 4.1 is repeated 20 times to obtain the graph of the variation of the distance between the two center points, as shown in Figure 10. As can be seen from Figure 10, the distance between the two center points gradually decreases with the increase of the number of experiments, which laterally reflects that the offset of the robot's moving trajectory is gradually

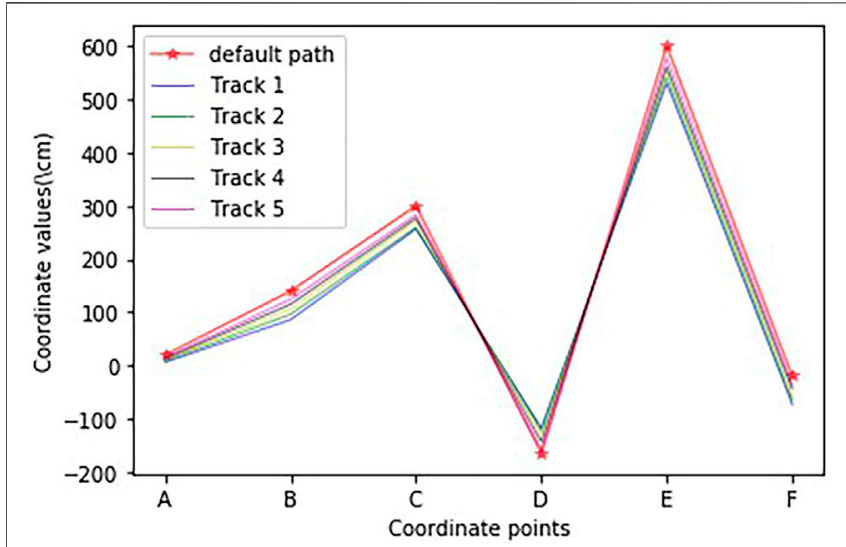

FIGURE 11 | Comparison of coordinate changes of the first five movement trajectories.

decreasing, thus improving the robot's moving accuracy and realizing the optimization of the robot's moving trajectory.

Further, to determine the location where the robot's trajectory deviation occurred, the first five experimental paths were selected, and the actual coordinates of six key points were recorded and compared with the preset key point coordinates. The results are shown in Figure 11, and the red pentagons are the coordinates of the preset path key points. From the overall change, the actual moving path gradually approximates the preset path as the number of experiments increases; In terms of local variation, the trajectory coordinates of points B, D, and E vary greatly. After analysis, the reason for this phenomenon may be that the friction force on the robot becomes larger during the lateral translation of the robot, resulting in the robot not reaching the preset key point.

\section{DISCUSSION}

In this paper, the trajectory optimization of mobile robots is achieved through digital twin technology. A mobile robot was designed, using the STM32 to control the movement of the robot and to receive data. A virtual model corresponding to a physical entity as well as a virtual environment that is the same as the real scene are created in Unity. Based on the data interaction characteristics of the digital twin technology, the robot's movement path is dynamically adjusted, the robot's movement accuracy is improved, and the closed-loop control combining virtual and actual is realized. The purpose of this work is not to innovate path planning algorithms, but to apply digital twin technology to mobile robots as an applied innovation. In contrast to the work done by others, this work focuses on the establishment of a digital twin of the mobile robot, the completion of the communication between the virtual model and the physical entity, and the realization of the trajectory optimization of the mobile robot.

This paper provides a reference for the application of digital twin technology in logistics and transportation industry, and 
provides a method to solve the connection between virtual models and physical entities, which is beneficial to promote the application of digital twin in other industries. However, the movement path of the robot in this paper is relatively simple and there are fewer obstacles on the movement path. In future work, it is necessary to consider how to improve the trajectory accuracy of the robot under more complex moving paths and to consider the time complexity and computational complexity of the algorithm. Meanwhile, the construction method of virtual model and the real time of real and virtual data interaction also need to be further studied.

\section{DATA AVAILABILITY STATEMENT}

The raw data supporting the conclusion of this article will be made available by the authors, without undue reservation.

\section{REFERENCES}

Bai, D., Sun, Y., Tao, B., Tong, X., Xu, M., Jiang, G., et al. (2021). Improved SSD Target Detection Method Based on Deep Feature Fusion. Concurrency Comput. Pract. Experience. doi:10.1002/CPE.6614

Chen, T., Peng, L., Yang, J., and Cong, G. (2021b). Analysis of User Needs on Downloading Behavior of English Vocabulary APPs Based on Data Mining for Online Comments. Mathematics 9 (12), 1341. doi:10.3390/math9121341

Chen, T., Rong, J., Yang, J., Cong, G., and Li, G. (2021a). Combining Public Opinion Dissemination with Polarization Process Considering Individual Heterogeneity. Healthcare 9 (2), 176. doi:10.3390/healthcare9020176

Chen, T., Yin, X., Peng, l., Rong, J., Yang, J., and Cong, G. (2021c). Monitoring and Recognizing Enterprise Public Opinion from High-Risk Users Based on User Portrait and Random Forest Algorithm. Axioms 10 (2), 106. doi:10.3390/axioms10020106

Cheng, Y., Li, G., Yu, M., Jiang, D., Yun, J., Liu, Y., et al. (2021). Gesture Recognition Based on Surface Electromyography -feature Image. Concurrency Computat Pract. Exper 33 (6), e6051. doi:10.1002/cpe.6051

Deng, X., Li, R., Zhao, L., Wang, K., and Gui, X. (2021). Multi-obstacle Path Planning and Optimization for mobile Robot. Expert Syst. Appl. 183, 115445. doi:10.1016/j.eswa.2021.115445

Duan, H., Sun, Y., Cheng, W., Jiang, D., Yun, J., Liu, Y., et al. (2021). Gesture Recognition Based on Multi-modal Feature Weight. Concurrency Computat Pract. Exper 33 (5), e5991. doi:10.1002/cpe.5991

Fu, Z., Wu, L., Qiao, T., Shi, B., and Yu, Q. (2021). Research on Obstacle Avoidance Algorithm of Handling Robot Based on Improved Artificial Potential Field Method. Machine Tools and Hydraulics 49, 25-29. doi:10.3969/j.issn.1001-3881.2021.05.005

Guo, H., Zhu, Y., Zhang, Y., Ren, Y., Chen, M., and Zhang, R. (2021). A Digital TwinBased Layout Optimization Method for Discrete Manufacturing Workshop. Int. J. Adv. Manuf Technol. 112, 1307-1318. doi:10.1007/s00170-020-06568-0

Hao, Z., Wang, Z., Bai, D., Tao, B., Tong, X., and Chen, B. (2021). Intelligent Detection of Steel Defects Based on Improved Split Attention Networks. Front. Bioeng. Biotechnol. doi:10.3389/fbioe.2021.810876

He, Y., Li, G., Liao, Y., Sun, Y., Kong, J., Jiang, G., et al. (2019). Gesture Recognition Based on an Improved Local Sparse Representation Classification Algorithm. Cluster Comput. 22 (Suppl. 5), 10935-10946. doi:10.1007/s10586-017-1237-1

Hu, J., Sun, Y., Li, G., Jiang, G., and Tao, B. (2019). Probability Analysis for Grasp Planning Facing the Field of Medical Robotics. Measurement 141, 227-234. doi:10.1016/j.measurement.2019.03.010

$\mathrm{Hu}$, W. (2021). Robot Obstacle Detection Method Based on Content Image Retrieval. Comput. Eng. Des. 42, 822-829. doi:10.16208/j.issn1000-7024.2021.03.032

Huang, L., Fu, Q., He, M., Jiang, D., and Hao, Z. (2021). Detection Algorithm of Safety Helmet Wearing Based on Deep Learning. Concurrency Computat Pract. Exper 33 (13), e6234. doi:10.1002/cpe.6234

\section{AUTHOR CONTRIBUTIONS}

All authors listed have made a substantial, direct, and intellectual contribution to the work and approved it for publication.

\section{FUNDING}

This research was supported by the National Natural Science Foundation of China (Nos. 52075530, 51575407, 51505349, 51975324, 61733011, and 41906177). The Grants of Hubei Provincial Department of Education (D20191105); The Grants of National Defense PreResearch Foundation of Wuhan University of Science and Technology (GF201705) and Open Fund of the Key Laboratory for Metallurgical Equipment and Control of Ministry of Education in Wuhan University of Science and Technology (2018B07 and 2019B13) and Open Fund of Hubei Key Laboratory of Hydroelectric Machinery Design and Maintenance in Three Gorges University (2020KJX02 and 2021KJX13).

Huang, L., Fu, Q., Li, G., Luo, B., Chen, D., and Yu, H. (2019). Improvement of Maximum Variance Weight Partitioning Particle Filter in Urban Computing and Intelligence. IEEE Access 7, 106527-106535. doi:10.1109/ACCESS.2019.2932144

Huang, L., He, M., Tan, C., Jiang, D., Li, G., and Yu, H. (2020). Jointly Network Image Processing: Multi-task Image Semantic Segmentation of Indoor Scene Based on CNN. IET image process 14 (15), 3689-3697. doi:10.1049/iet-ipr.2020.0088

Huo, G., and Wang, X. (2021). Analysis and Research on Control Algorithm of Logistics Handling Robot Based on Agricultural Warehouse. Chin. J. Construction Machinery 19, 56-60. doi:10.15999/j.cnki.311926.2021.01.010

Hur, S. W., Lee, S. H., Nam, Y. H., and Kim, C.-J. (2021). Direct DynamicSimulation Approach to Trajectory Optimization. Chin. J. Aeronautics 34, 6-19. doi:10.1016/j.cja.2021.01.019

Jiang, D., Li, G., Sun, Y., Hu, J., Yun, J., and Liu, Y. (2021a). Manipulator Grabbing Position Detection with Information Fusion of Color Image and Depth Image Using Deep Learning. J. Ambient Intell. Hum. Comput 12 (12), 10809-10822. doi:10.1007/s12652-020-02843-w

Jiang, D., Li, G., Sun, Y., Kong, J., Tao, B., and Chen, D. (2019b). Grip Strength Forecast and Rehabilitative Guidance Based on Adaptive Neural Fuzzy Inference System Using sEMG. Pers Ubiquit Comput. doi:10.1007/s00779-019-01268-3

Jiang, D., Li, G., Sun, Y., Kong, J., and Tao, B. (2019a). Gesture Recognition Based on Skeletonization Algorithm and CNN with ASL Database. Multimed Tools Appl. 78 (21), 29953-29970. doi:10.1007/s11042-018-6748-0

Jiang, D., Li, G., Tan, C., Huang, L., Sun, Y., and Kong, J. (2021b). Semantic Segmentation for Multiscale Target Based on Object Recognition Using the Improved Faster-RCNN Model. Future Generation Comput. Syst. 123, 94-104. doi:10.1016/j.future.2021.04.019

Jiang, D., Zheng, Z., Li, G., Sun, Y., Kong, J., Jiang, G., et al. (2019c). Gesture Recognition Based on Binocular Vision. Cluster Comput. 22 (Suppl. 6), 13261-13271. doi:10.1007/s10586-018-1844-5

Jones, D., Snider, C., Nassehi, A., Yon, J., and Hicks, B. (2020). Characterising the Digital Twin: A Systematic Literature Review. CIRP J. Manufacturing Sci. Tech. 29, 36-52. doi:10.1016/j.cirpj.2020.02.002

Li, B., Sun, Y., Li, G., Kong, J., Jiang, G., Jiang, D., et al. (2019c). Gesture Recognition Based on Modified Adaptive Orthogonal Matching Pursuit Algorithm. Cluster Comput. 22 (Suppl. 1), 503-512. doi:10.1007/s10586-017-1231-7

Li, C., Li, G., Jiang, G., Chen, D., and Liu, H. (2020). Surface EMG Data Aggregation Processing for Intelligent Prosthetic Action Recognition. Neural Comput. Applic 32 (22), 16795-16806. doi:10.1007/s00521-018-3909-z

Li, G., Jiang, D., Zhou, Y., Jiang, G., Kong, J., and Manogaran, G. (2019b). Human Lesion Detection Method Based on Image Information and Brain Signal. IEEE Access 7, 11533-11542. doi:10.1109/ACCESS.2019.2891749

Li, G., Li, J., Ju, Z., Sun, Y., and Kong, J. (2019a). A Novel Feature Extraction Method for Machine Learning Based on Surface Electromyography from Healthy Brain. Neural Comput. Applic 31 (12), 9013-9022. doi:10.1007/s00521-019-04147-3s 
Liao, S., Li, G., Wu, H., Jiang, D., Liu, Y., Yun, J., et al. (2021). Occlusion Gesture Recognition Based on Improved SSD. Concurrency Comput. Pract. Experience 33 (6), e6063. doi:10.1002/cpe.6063

Liu, J., Cao, X., Zhou, H., Li, L., Liu, X., Zhao, P., et al. 2021). A Digital TwinDriven Approach towards Traceability and Dynamic Control for Processing Quality. Adv. Eng. Inform. 50, 101395. do, doi:10.1016/j.aei.2021.101395

Liu, Q., Leng, J., Yan, D., Zhang, D., Wei, L., Yu, A., et al. (2021). Digital TwinBased Designing of the Configuration, Motion, Control, and Optimization Model of a Flow-type Smart Manufacturing System. J. Manufacturing Syst. 58, 52-64. doi:10.1016/j.jmsy.2020.04.012

Liu, Y., Jiang, D., Duan, H., Sun, Y., Li, G., Tao, B., et al. (2021a). Dynamic Gesture Recognition Algorithm Based on 3D Convolutional Neural Network. Comput. Intelligence Neurosci. 2021, 1-12. doi:10.1155/2021/4828102

Liu, Y., Jiang, D., Tao, B., Qi, J., Jiang, G., Yun, J., et al. (2021c). Grasping Posture of Humanoid Manipulator Based on Target Shape Analysis and Force Closure. Alexandria Eng. J. doi:10.1016/j.aej.2021.09.017

Liu, Y., Li, C., Jiang, D., Chen, B., Sun, N., Cao, Y., et al. (2021a). Wrist Angle Prediction under Different Loads Based on GA-ELM Neural Network and Surface Electromyography. Concurrency Computat Pract. Exper. doi:10.1002/CPE.6574

Liu, Y., Xiao, F., Tong, X., Tao, B., Xu, M., Jiang, G., et al. (2021b). Manipulator Trajectory Planning Based on Work Subspace Division. Concurrency Comput. Pract. Experience. doi:10.1002/cpe.6710

Liu, Y., Xu, M., Jiang, G., Tong, X., Yun, J., Liu, Y., et al. (2021b). Target Localization in Local Dense Mapping Using RGBD SLAM and Object Detection. Concurrency Computat Pract. Exper. doi:10.1002/cpe.6655

Liu, Y., Jiang, D., Yun, J., Sun, Y., Li, C., Jiang, G., et al. (2021d). Self-Tuning Control of Manipulator Positioning Based on Fuzzy PID and PSO Algorithm. Front. Bioeng. Biotechnol. doi:10.3389/fbioe.2021.817723

Lu, Y., Liu, C., Wang, K. I.-K., Huang, H., and Xu, X. (2020). Digital Twin-Driven Smart Manufacturing: Connotation, Reference Model, Applications and Research Issues. Robotics and Computer-Integrated Manufacturing 61, 101837. doi:10.1016/j.rcim.2019.101837

Luo, B., Sun, Y., Li, G., Chen, D., and Ju, Z. (2020). Decomposition Algorithm for Depth Image of Human Health Posture Based on Brain Health. Neural Comput. Applic 32 (10), 6327-6342. doi:10.1007/s00521-019-04141-9

Ma, R., Zhang, L., Li, G., Jiang, D., Xu, S., and Chen, D. (2020). Grasping Force Prediction Based on sEMG Signals. Alexandria Eng. J. 59 (3), 1135-1147. doi:10.1016/j.aej.2020.01.007

Matulis, M., and Harvey, C. (2021). A Robot Arm Digital Twin Utilising Reinforcement Learning. Comput. Graphics 95, 106-114. doi:10.1016/j.cag.2021.01.011

Miao, C., Chen, G., Yan, C., and Wu, Y. (2021). Path Planning Optimization of Indoor mobile Robot Based on Adaptive Ant colony Algorithm. Comput. Ind. Eng. 156, 107230. doi:10.1016/j.cie.2021.107230

Nazarahari, M., Khanmirza, E., and Doostie, S. (2019). Multi-objective MultiRobot Path Planning in Continuous Environment Using an Enhanced Genetic Algorithm. Expert Syst. Appl. 115, 106-120. doi:10.1016/j.eswa.2018.08.008

Niloy, M. A. K., Shama, A., Chakrabortty, R. K., Ryan, M. J., Badal, F. R., Tasneem, Z., et al. (2021). Critical Design and Control Issues of Indoor Autonomous Mobile Robots: A Review. IEEE ACCESS 9, 35338-35370. doi:10.1109/ ACCESS.2021.3062557

Petrović, L., Peršić, J., Seder, M., and Marković, I. (2020). Cross-entropy Based Stochastic Optimization of Robot Trajectories Using Heteroscedastic Continuous-Time Gaussian Processes. Robotics Autonomous Syst. 133, 103618. doi:10.1016/j.robot.2020.103618

Ruzsa, C. (2021). Digital Twin Technology - External Data Resources in Creating the Model and Classification of Different Digital Twin Types in Manufacturing. Proced. Manufacturing 54, 209-215. doi:10.1016/j.promfg.2021.07.032

Sarkar, R., Barman, D., and Chowdhury, N. (2020). Domain Knowledge Based Genetic Algorithms for mobile Robot Path Planning Having Single and Multiple Targets. J. King Saud Univ. - Comput. Inf. Sci. doi:10.1016/j.jksuci.2020.10.010

Sun, Y., Hu, J., Li, G., Jiang, G., Xiong, H., Tao, B., et al. (2020c). Gear Reducer Optimal Design Based on Computer Multimedia Simulation. J. Supercomput 76 (6), 4132-4148. doi:10.1007/s11227-018-2255-3

Sun, Y., Tian, J., Jiang, D., Tao, B., Liu, Y., Yun, J., et al. (2020b). Numerical Simulation of thermal Insulation and Longevity Performance in New Lightweight Ladle. Concurrency Computat Pract. Exper 32 (22), e5830. doi: $10.1002 /$ cpe. 5830
Sun, Y., Weng, Y., Luo, B., Li, G., Tao, B., Jiang, D., et al. (2020a). Gesture Recognition Algorithm Based on Multi-scale Feature Fusion in RGB-D Images. IET image process 14 (15), 3662-3668. doi:10.1049/iet-ipr.2020.0148

Sun, Y., Xu, C., Li, G., Xu, W., Kong, J., Jiang, D., et al. (2020d). Intelligent Human Computer Interaction Based on Non Redundant EMG Signal. Alexandria Eng. J. 59 (3), 1149-1157. doi:10.1016/j.aej.2020.01.015

Sun, Y., Yang, Z., Tao, B., Jiang, G., Hao, Z., and Chen, B. (2021). Multiscale Generative Adversarial Network for Real-world Super-resolution. Concurrency Computat Pract. Exper 33. doi:10.1002/CPE.6430

Tan, C., Sun, Y., Li, G., Jiang, G., Chen, D., and Liu, H. (2020). Research on Gesture Recognition of Smart Data Fusion Features in the IoT. Neural Comput. Applic 32 (22), 16917-16929. doi:10.1007/s00521-019-04023-0

Tao, B., Liu, Y., Huang, L., Chen, G., and Chen, B. (2021). 3D Reconstruction Based on Photoelastic Fringes. Concurrency Computat. Pract. Exper. 34 (1), e6481. doi:10.1002/cpe.6481

Tao, F., Liu, W., Liu, J., Liu, X., Liu, Q., Qu, T., et al. (2018). Digital Twin and its Application Exploration. Comput. Integrated Manufacturing Syst. 24, 1-18. doi:10.13196/j.cims.2018.01.001

Tao, F., Liu, W., Zhang, M., Hu, T., Qi, Q., Zhang, H., et al. (2019). Digital Twin Five-Dimensional Model and Ten Domain Applications. Comput. Integrated Manufacturing Syst. 25, 1-18. doi:10.13196/j.cims.2019.01.001

Verner, I., Cuperman, D., Fang, A., Reitman, M., Romm, T., and Balikin, G. (2018). Robot Online Learning Through Digital Twin Experiments: A Weightlifting Project. Online Eng. Internet Thing, 307-314. doi:10.1007/978-3-319-64352-6_29

Weng, Y., Sun, Y., Jiang, D., Tao, B., Liu, Y., Yun, J., et al. (2021). Enhancement of Real-Time Grasp Detection by Cascaded Deep Convolutional Neural Networks. Concurrency Comput. Pract. Experience 33 (5), e5976. doi:10.1002/cpe.5976

Xiao, F., Li, G., Jiang, D., Xie, Y., Yun, J., Liu, Y., et al. (2021). An Effective and Unified Method to Derive the Inverse Kinematics Formulas of General SixDOF Manipulator with Simple Geometry. Mechanism Machine Theor. 159, 104265. doi:10.1016/j.mechmachtheory.2021.104265

Yang, Z., Jiang, D., Sun, Y., Tao, B., Tong, X., Jiang, G., et al. (2021). Dynamic Gesture Recognition Using Surface EMG Signals Based on Multi-Stream Residual Network. Front. Bioeng. Biotechnol. 9. doi:10.3389/fbioe.2021.779353

Yu, M., Li, G., Jiang, D., Jiang, G., Tao, B., and Chen, D. (2019). Hand Medical Monitoring System Based on Machine Learning and Optimal EMG Feature Set. Pers Ubiquit Comput. doi:10.1007/s00779-019-01285-2

Yun, J., Sun, Y., Li, C., Jiang, D., Tao, B., Li, G., et al. (2021). Self-adjusting Force/Bit Blending Control Based on Quantitative Factor-Scale Factor Fuzzy-PID Bit Control. Alexandria Eng. J. doi:10.1016/j.aej.2021.09.067

Zhao, G., Jiang, D., Liu, X., Tong, X., Sun, Y., Tao, B., et al.(2021). A Tandem Robotic Arm Inverse Kinematic Solution Based on an Improved Particle Swarm Algorithm. Front. Bioeng. Biotechnol. doi:10.3389/fbioe.2021.832829

Zheng, K., Wu, F., and Chen, X. (2021). Laser-Based People Detection and Obstacle Avoidance for a Hospital Transport Robot. Sensors 21, 961. doi:10.3390/s21030961

Zhuang, C., Liu, J. H., Xiong, H., Ding, X., Liu, S., and Wen, G. (2017). The Connotation, Architecture and Development Trend of Product Digital Twin. Comput. Integrated Manufacturing Syst. 23, 753-768. doi:10.13196/j.cims.2017.04.010

Conflict of Interest: The authors declare that the research was conducted in the absence of any commercial or financial relationships that could be construed as a potential conflict of interest.

Publisher's Note: All claims expressed in this article are solely those of the authors and do not necessarily represent those of their affiliated organizations, or those of the publisher, the editors, and the reviewers. Any product that may be evaluated in this article, or claim that may be made by its manufacturer, is not guaranteed or endorsed by the publisher.

Copyright (C) 2022 Liu, Jiang, Tao, Jiang, Sun, Kong, Tong, Zhao and Chen. This is an open-access article distributed under the terms of the Creative Commons Attribution License (CC BY). The use, distribution or reproduction in other forums is permitted, provided the original author(s) and the copyright owner(s) are credited and that the original publication in this journal is cited, in accordance with accepted academic practice. No use, distribution or reproduction is permitted which does not comply with these terms. 